\title{
Tangence
}

\section{Le festivalesque (La ville dans le roman de Réjean Ducharme)}

\section{Pierre Popovic}

Numéro 48, octobre 1995

Montréal et Vancouver : parcours urbains dans la littérature et le cinéma

URI : https://id.erudit.org/iderudit/025866ar

DOI : https://doi.org/10.7202/025866ar

Aller au sommaire du numéro

Éditeur(s)

Tangence

ISSN

0226-9554 (imprimé)

1710-0305 (numérique)

Découvrir la revue

Citer cet article

Popovic, P. (1995). Le festivalesque (La ville dans le roman de Réjean

Ducharme). Tangence, (48), 116-127. https://doi.org/10.7202/025866ar d'utilisation que vous pouvez consulter en ligne.

https://apropos.erudit.org/fr/usagers/politique-dutilisation/ 


\section{Le festivalesque (La ville dans le roman de Réjean Ducharme) \\ Pierre Popovic}

J'ai eu raison dans tous mes dédains: puisque je m'évade! Je m'évade!

Rimbaud, “ L'Impossible * 1

Sous l'impulsion d'André Belleau notamment, la critique québécoise a réservé un accueil plus que chaleureux aux ouvrages de Mikhail Bakhtine, particulièrement à ses deux célèbres essais sur la cueillaison de la tradition comique populaire dans l'œuvre de François Rabelais ${ }^{2}$ et sur l'esthétique romanesque de Dostö̈evski ${ }^{3}$. Outre l'intérêt intrinsèque et immédiatement théorique de la critique bakhtinienne, plusieurs raisons, reliées à un désir de renouvellement des lectures établies de la littérature québécoise, expliquent l'attention qu'elle reçut, dès la fin des années soixante-dix et au cours des années quatre-vingt, dans la Belle Province. Bien qu'il soit évident qu'elles sont entremêlées, puisque tous nos choix critiques, quelle que soit la vigueur de leur aspiration à la distance et à l'objectivité, doivent une part de leur être à l'air du temps, aux circonstances, j'énumérerai et distinguerai trois de ces raisons.

La première est une cause circonstancielle et stratégique. Basée sur une idée à ce point haute de la démocratie qu'elle en oublie de tenir compte des antagonismes entre les groupes sociaux et des incompatibilités et rivalités entre les intérêts privés, fondée sur une conception impérativement pluraliste et du jeu social et des jeux littéraires, l'herméneutique bakhtinienne convient à merveille à une instance critique décidée à renforcer à sa

1 Arthur Rimbaud, "L'Impossible", Une saison en enfer, dans Arthur Rimbaud, Euvre-Vie. Édition du Centenaire établie par Alain Borer, Paris, Arléa, 1991, p. 439.

2 Mikhaïl Bakhtine, L'cuvre de François Rabelais et la culture populaire au Moyen Âge et sous la Renaissance, Paris, Gallimard, coll. "Tel", 1982, 471 p.

3 Mikhail Bakhtine, La poétique de Dostoïeuski, Paris, Seuil, coll. "Pierres vives", $1970,346 \mathrm{p}$. 
façon l'évidence socio-politique vite consacrée par la doxa d'un passage radical de la Grande Noirceur à la Révolution tranquille, de la communauté refermée sur elle-même à son ouverture au monde, du passéisme clérical au progrès läque, de la tradition à la modernité, etc. ${ }^{4}$

La seconde raison est proprement heuristique. Les couplages notionnels qui opposent chez Bakhtine l'homogénéité à l'hétérogénéité, l'un au multiple, l'autorité à la vie débordante, le monologisme à la variété, etc., selon une série de binômes paradigmatiques qui trouvent leur parangon symbolique dans l'opposition entre le discours officiel et la culture populaire (le camaval), et leur parangon politique dans l'opposition entre le totalitarisme et la liberté, ont constitué des outils heuristiques aptes à rendre compte de nouveaux textes et de nouveaux styles et, du même coup, à relire certains textes du passé à l'aune de ce que le nationalisme avait refusé d'y voir, à savoir tout ce qui relève du multi-, du pluriet de l'hétéro-, de l'étrangeté et de l'étrangèreté: plurilinguismes, compénétrations stylistico-culturelles, intrications des styles nobles et burlesques ${ }^{5}$, conflits de $\operatorname{codes}^{6}$, hétéroglossies, etc. ${ }^{7}$

La troisième raison se résume à un nom: Montréal. Montréal, non comme "destination irréaliste" comme le pense un personnage de L'biver de Mira Christophe, mais comme lieu par excellence des mélanges, des mosaiqques et des bigarrures, acmé des hétéromorphies et des croisements en tout genre, arène des concurrences, des modes, des mouvements, des conflits, des embras-

4 Dans la perspective d'une histoire critique de la critique, le point de vue pourrait être acéré et il serait tout à fait concevable de soutenir qu'il y eut une lecture singulière de Bakhtine, partielle et sélective, intégrant certains éléments des travaux de ce dernier dans ce que Jocelyn Létourneau, prenant justement à parti les évidences socio-politiques dont il est question ci-dessus, appelle le récit collectif du "Québec moderne". Cf. Jocelyn Létourneau, "Le "Québec moderne" : un chapitre du grand récit collectif des Québécois ", Discours social/Social Discourse, vol. 4, n ${ }^{\text {os }} 1-2$, hiver-printemps 1992, p. 63-88.

5 Voir à ce sujet plusieurs des études que Pierre Nepveu a rassemblées dans L'écologie du réel, Montréal, Boréal, 1988, 243 p.

6 Selon les termes utilisés par André Belleau, "Le conflit des codes dans l'institution littéraire québécoise", in Surprendre les voix, Montréal, Boréal, 1986, $237 \mathrm{p}$.

7 Soit dit en passant, il est curieux (mais compréhensible) que la question du rapport entre les cultures populaire et savante au Québec, problématique pourtant jugée névralgique et capitale par André Belleau, ait été aussi peu soulevée depuis la disparition de ce dernier. 
118

sements et des embrasements, des marges et des déviances, Montréal "grand désordre universel" comme l'estampille son plus grand poète, la ville telle qu'en elle-même toutes les modernités et les contemporanéités la changent, la ville creuset ou relais de tous les discours et productrice elle-même de ce discours social qui la légitime et nous la représente sans fin, productrice indirecte et à temps partiel de ces écritures littéraires qui sont traversées par ce discours social, écritures littéraires qui - je referme la boucle - semblent, en raison même de leur lieu d'émergence, si bien se prêter à des études bakhtiniennes.

L'une des notions bakhtiniennes les plus employées à propos de la littérature québécoise, ou plus exactement à propos du roman montréalais, est celle de carnavalesque ou de carnavalisation. Des écrits de Gilbert LaRoque, Hubert Aquin, Michel Tremblay, Jacques Ferron, Yolande Villemaire, et de bien d'autres, ont été dits carnavalesques. Or, il me semble que l'on est allé parfois un peu vite en besogne dans l'application immédiate des outils notionnels et conceptuels bakhtiniens. Dans le cas précis du carnavalesque, je pense que cette qualification ne rend que très partiellement compte des écritures romanesques montréalaises récentes. J'entends le montrer en m'intéressant à l'un des romanciers les plus mystérieusement et typiquement montréalais, Réjean Ducharme lui-même, et m'appuyer sur cette démonstration pour proposer un outil de description plus approprié à ce que sont l'esthétique ducharmienne, prise ici à titre d'exemple, et d'autres proses narratives montréalaises.

Le transfert brutal, sans discussion et sans aménagement, de certains concepts bakhtiniens sur la littérature moderne pose en effet de sérieux problèmes dont le moindre n'est pas que l'inscription socio-historique des textes disparait généralement dans de semblables utilisations. Car, quand bien même arriverait-on à prouver qu'il y a du carnavalesque chez Hubert Aquin, il reste que le sens de cette éventuelle carnavalisation de l'écriture ne saurait être le même chez l'auteur de Procbain épisode et chez le renaissant Rabelais. C'est d'ailleurs Bakhtine lui-même qui invite à la plus grande circonspection, par exemple quand il épingle l'affadissement de la parodie moderne dans Esthétique et théorie $d u r_{m a n}{ }^{8}$, quand il distingue radicalement " le grotesque roman-

8 Mikhaïl Bakhtine, Esthêtique et théorie du roman, Paris, Gallimard, coll. "Bibliothèque des idées", 1978, 488 p. 
tique "(qui n'est qu'un repoussoir permettant à des écrivains comme Hugo d'asseoir la catégorie du sublime) et "l'imagerie grotesque de la culture populaire du Moyen Âge et de la Renaissance" 9 , ou encore quand il décrit à plusieurs reprises au cours de l'"Introduction" de son Rabelais la dégradation du carnaval à l'heure moderne ${ }^{10}$.

On objectera que Bakhtine cite quelques écrivains du $\mathrm{xx}^{\mathrm{e}}$ siècle et invite à les situer dans la lignée du carnavalesque ${ }^{11}$. Mais justement: il les place dans la foulée de, dans la suite de, il en fait des héritiers, non des fidèles, insistant bien sur les différences entre leurs esthétiques et le carnavalesque des soties ou du Gargantua, notant que chez eux "tous les motifs principaux tirant manifestement leur origine du carnaval conservent une certaine réminiscence de l'ensemble puissant dont ils constituaient jadis des parcelles " 12 . Il y a donc pour Bakhtine des résonances du carnavalesque à l'heure moderne, des éclats, des résidus, des fragments d'énergie issus de ce big bang narratif que fut le comique populaire dans l'imaginaire médiéval et chez Rabelais. Mais rien de plus. Dès lors, la question qui se pose est la suivante: quelles sont les possibles rémanences du carnavalesque dans les littératures modernes?

Tournant autour d'une question semblable, Pierre V. Zima écrit que "Dans le cas de la culture et de la littérature modernes,

9 Mikhaïl Bakhtine, L'cuvre de François Rabelais..., p. 49 et $s$.

10 La distinction entre le carnavalesque médiéval et renaissanciste et le "grotesque moderniste" est posée dans les termes les plus forts au cours de la discussion que fait Bakhtine du livre de Wolfgang Kayser, Das Groteske in Malerei und Dichtung. Bakhtine écrit notamment: "Kayser souligne particulièrement l'aspect étranger: "Le grotesque est un monde devenu étranger" ("das Groteske ist die entfremdete Welt" [...]). Il explique cette définition en comparant le grotesque à l'univers des contes. Celui-ci, vu de l'extérieur, peut aussi être défini comme étranger et insolite, mais ce n'est pas un monde devenu extérieur. Dans le grotesque, par contre, ce qui pour nous était à nous, proche, devient soudain extérieur et hostile. C'est notre monde qui se transforme soudain en monde des autres. Cette définition, applicable à certains phénomènes du grotesque moderne, n'est pas entièrement appropriée au grotesque romantique, et plus du tout aux phases antérieures." (Ibid., p. 57).

11 Il s'agit de Jarry, des surréalistes et des expressionnistes qu'il rattache au "grotesque modemiste ", et de Thomas Mann, Bertolt Brecht et Pablo Neruda qu'il rattache au grotesque réaliste.

12 Ibid., p. 56. Aux yeux du critique russe, le seul dont l'œuvre "reflète[rait...] l'influence directe des formes carnavalesques" est Pablo Neruda. 
"carnaval" devient une désignation métonymique de "marché"13. En fait, c'est trop peu dire. Car entre le carnaval dont Bakhtine fait l'Urform d'une esthétique romanesque historiquement située et le carnaval exsangue, folklorique, touristique et commercial du $x^{e}{ }^{e}$ siècle, le changement ne se résume pas à un glissement métonymique. Un aspect décisif du carnavalesque disparaît, surtout si l'on entend bien le mot marchê au sens contemporain, économique et libéral, et en le dégageant du côté "sympathique et bruyant assemblage d'étals sur une place publique "qu'il conserve encore quelquefois dans les prospectus touristiques: si le multiple et la pluralité des voix se maintiennent, s'ils se généralisent même et atteignent des indices de croissance exponentiels, ils sont cependant soumis à des lois et des logiques autrement plus sévères que celles des places publiques d'antan; ces lois nouvelles sont celles des déséquilibres entre l'offre et la demande, des perpétuels états de crise, des concurrences les plus dures, du dumping social et des publicités agressives, des "rationalisations" et des faillites provoquées, des liquidations des stocks et des ventes "en spécial", des chômages sectoriels planifiés et des soldes du jour sur grande surface, des entreposages de déchets, des recyclages et des récupérations, des marchandises jetables après usage, des nouvelles pauvretés et des abaissements des prix de revient des produits par attrition naturelle de la main d'œuvre productrice (sic! entendu tel quel dans une émission radiophonique économique), etc. C'est cela la ville contemporaine en tant que marché. Ce qui demeure, du point de vue d'un carnavalesque potentiel qui serait la contrepartie esthétique de ce que je viens de décrire, c'est essentiellement la pluralité des langues, des styles, des us et des coutumes, mais deux choses ont résolument disparu à l'égard du carnavalesque renaissant: d'une part le rire du corps débordant de santé, le rire de fête célébré par Rabelais relu par Bakhtine, d'autre part le renversement du haut par le bas, le bouleversement des hiêrarchies, l'inversion de l'auctoritas et de ses attributs symboliques, le chambardement de "l'ordre" et tout ce qui relève de la petite révolution ritualisée, de la régénérescence festoyante et luxuriante. Ces deux éléments sont à ce point fondamentaux que parler encore de carnavalisation ou de carnavalesque à propos d'écritures contemporaines me paraît délicat.

13 Pierre V. Zima, Manuel de sociocritique, Paris, Picard, 1985, p. 110. 
J'ai ces réflexions en tête, et je me promène à la fois dans Montréal et dans l'œuvre de Réjean Ducharme, et je me demande, sachant bien qu'il y a chez l'auteur de L'océantume de ces plurilinguismes, de ces télescopages de sociolectes et de ces hétéroglossies qui sont des échos du carnavalesque triomphant, je me demande s'il n'est pas possible de trouver entre la ville et le roman une analogie, suffisamment parlante pour n'être pas qu'approximative et suffisamment souple pour n'être pas de l'ordre du simple reflet, une analogie qui puisse qualifier au mieux la prise réciproque de Montréal par le roman ducharmien.

L'incipit de L'biver de force présente les deux personnages principaux du roman, les presque trentenaires "bommes de Maskinongé " André et Nicole, vivant enfermés dans leur appartement comme dans une boîte de sardines, reliés à l'extérieur par leur télévision, le téléphone, quelques "amis" et, surtout, par "l'anathème, les potins, les farces" dont ils usent et abusent afin de ne pas se laisser déposséder d'eux-mêmes, afin de pouvoir exprimer leur "haine pour tout ce qui veut [les] faire vouloir comme des dépossédés" ${ }^{14}$. Ces railleries, rosseries, égratignures, coups de griffe, cette malice ironique, ces persiflages moqueurs, cette méchanceté forcenée et constante, quasiment thérapeutique, sont le moyen qu'ils ont trouvé afin de résister encore et toujours à l'envahisseur, afin de ne pas se laisser enrégimenter par les vendeurs d'espoir de tout acabit ${ }^{15}$. La ville est extérieure aux personnages et le principal courant d'air qui va de l'une aux autres est constitué par des informations télévisuelles qui jouent ici le rôle d'un cordon ombilical technologique et froid; elle n'est perçue qu'avec dédain, se résume à une foire de mots creux, de séductions illusoires, ringardes et nigaudes (les livres, les films, les idées "de tous ceux qui nous aiment"), à une immense machine à fabriquer de fausses et dangereuses croyances. C'est d'ailleurs parce que l'atmosphère commence à leur paraitre paranoïde qu'ils décident de changer de cap — "ça n'a plus de bon sens" - de désormais demeurer sourds à tout le bruit urbain, de s'en tenir à ce qu'ils veulent que le réel soit et non au réel médiatisé que la ville propose: "dire que c'est ça qui est ça puis aller

14 Réjean Ducharme, L'biver de force, Paris, Gallimard, coll. "Folio ", 1990, p. 16.

15 Tous ceux qui " veulent absolument que nous quittions l'angoisse de nos chaises pour nous embarquer dans leur jumbo-bateau garanti tout confort jusqu'à la prochaine nouvelle vague (ibid., p. 15). 
jusqu'au bout " ${ }^{16}$. Pour célébrer cette décision et la nouvelle vie qui l'accompagne, vie dont André notera les événements avec sa belle écriture, ils organisent une petite fête dont la splendeur est évaluée à la présence de bière et de rhum White Sails, à "cinq boîtes de panatelas Garcia y Vega ", à "deux gros médaillons de filet de bœuf [qui leur ont coûté] $\$ 1,87$, ça fait que ça a besoin d'être bon", à l'achat d'un billet de Mini-Loto, le numéro 77706 (l'ironie de Ducharme ne recule devant nul réalisme), et à l'acquisition de "quatre fascicules de l'encyclopédie Alpha (la Mémoire du Temps), pour ne pas avoir l'air de profiter de ce qu'ils donnent les deux premiers pour le prix d'un "17. Tout cela est là "pour fêter notre décision de repartir à zéro", pour "inaugurer" comme on le fait lors des manifestations culturelles par un spectacle d'ouverture.

Inaugurer une vie nouvelle, repartir à zéro, c'est à cela que servait le carnaval au temps de toutes les traditions, augurant du renouveau général par sa période de bombance et de folie. Hormis ce minuscule point commun, rien dans cet incipit n'évoque de près ou de loin le carnavalesque. Qu'y découvre-t-on vraiment? Une dimension festive; la clôture d'un groupe réuni par son amitié amoureuse certes mais aussi par son dédain et son ironie à l'égard des pratiques urbaines extérieures; une accumulation de simulacres et de succédanés, les uns connotant l'aisance, l'abondance, la richesse, les autres le savoir, l'histoire, la culture; le désir de faire comme s'il était possible de "repartir à zéro", désir dont on sait par avance qu'il est illusoire et que son illusion ne durera que quelques moments ${ }^{18}$; enfin une forte présence de la valeur marchande des choses agrémentée de la joie de profiter des soldes temporaires. L'idée d'un renversement hiérarchique ritualisé est totalement étrangère à cette entreprise. Ce n'est pas à un carnaval que celle-ci fait penser, mais à un autre type de

$16 \quad$ Ibid., p. 17.

17 Ibid., p. 17-18.

18 Il y aurait une belle recherche à mener sur le rôle et la nature de la fête chez Ducharme. Ses romans sont parsemés de fêtes vaguement ringardes comparables à celle de l'incipit de L'biver de force. J'insiste ici sur le caractère très circonscrit dans le temps que possède cette fête. On en trouvẹ la confirmation et un autre exemple dans cette orgie de sandwiches au fromage kraft en tranches par laquelle André et Nicole compensent leur déception de ne pas avoir attiré l'attention de Petit Pois. Le narrateur résume ainsi le but poursuivi : "Durer à tout prix jusqu'à demain, jusqu'après avoir dormi. * 
manifestation socio-culturelle, dont la ville de Montréal fait une consommation ostentatoire et boulimique : le festival.

Quel que soit le moment de son arrivée, et même s'il éprouve une forte affection pour le grand festival de Bayreuth, tout étranger qui arrive à Montréal ne peut qu'en être surpris et stupéfait: Montréal n'existe que de façon quantique, de sursaut d'énergie en sursaut d'énergie ou, pour le dire dans les termes mêmes de la rumeur urbaine, Montréal n'existe que de festival en festival. Aux côtés du festival international du cinéma, du festival international de jazz ou du festival du théâtre des Amériques ${ }^{19}$, d'autres festivals plus communs fleurissent au gré des devantures et des saisons, à l'exemple des festivals des fèves au lard, des crevettes, du homard, de l'informatique, du camping sauvage ou de la chanson populaire. N'espérez ni faire des affaires ni oeuvrer dans le culturel à Montréal si vous ne pensez pas festival, si vous ne créez pas de festival, si vous ne possédez pas votre festival, Il y en a pour tous les goûts, de haut en bas de la gamme des habitus, et de toutes les sortes. Les "festivals-carrefours" des théâtres d'intervention, les festivals de la bière en fût, les festivals du Vieux-Port, les "festivals-santé", les festivals paroissiaux de la chanson catholique (il en reste) prolifèrent à l'ombre du très populaire festival "Juste pour rire " ${ }^{20}$. Bien entendu, le festival du REER de la banque du coin n'est jamais très loin de celui du rire, et telle marque réputée de jeans y est allée d'un jeu de signifiant digne de la revue TXT ou d'André Gervais, ornant maints panneaux publicitaires d'un affriolant "Fesse-tival". Dans le domaine alimentaire particulièrement, la moindre gargotte serait déshonorée de ne pas avoir son festival, en serait-il un du smoked-meat ou de la bière d'épinette.

Or qu'est-ce qu'un festival? C'est un moment surabondant et suractif de représentations comparables auxquelles sont conviés les adeptes d'une spécialité humaine, culturelle, alimentaire, comportementale, philosophique. C'est une façon de concilier le festif et le mercantile, la consommation et le culturel, la consommation de la culture et la culture de la consommation, le populaire et le

19 La liste de ces grosses manifestations pourrait être plus longue.

20 D'une notoriété telle qu'il a dernièrement débouché sur des projets aussi farfelus que la création d'un Musée du rire et d'une École internationale du rire, lesquels n'ont dô qu'à d'obscures circonstances de ne pas nous rendre définitivement risibles. 
124

sériel, l'abondance et la performance, le capitalisme de séduction et le spiritualisme du partage d'un sentiment ou d'un goût commun. Le festival est une mystique matérialiste de l'événement; il faut pouvoir dire "j'y étais" comme lors des grands festivals pop des années soixante, Woodstock ou Wight. Le festival, c'est la face noble du corporatisme ou de la spécialisation. C'est une façon de régir le charme, de gérer la jouissance, c'est un moment de fausse évasion temporaire, un dépaysement sans bouger, où l'on peut rester absolument individuel, comblé même dans l'individualisme de son goût, et où l'on communie pourtant avec une communauté élective soudainement vivante, présente, recomposée. C'est une régression moderne du moderne, une échappatoire au rabais mais sublime, où certaines règles sont suspendues réunion d'énormes assemblées, arrêt de la circulation routière, tapages semi-nocturnes autorisés, abus d'un produit exclusif, libation publique -, mais où le discours officiel et l'ordre ne sont pas remis en question, si ce n'est de façon purement plastique et clean $^{21}$.

Un moment de fausse évasion disais-je, autrement dit: une dévasion. Dévadé, dont le héros Bottom porte un nom ${ }^{22}$ qui semble faire appel à ce renversement du haut par le bas qui était la marque du carnavalesque mais dont le comportement et tout le roman montrent qu'il est à mille lieues de renverser quoi que ce soit (si ce n'est par la force d'une insistante et dédaigneuse inertie essentiellement bibitive ${ }^{23}$ ), est le prototype même de l'écriture festivalesque. Le style de Ducharme exhibe cette scansion quantique, cette accélération par grumeaux, ces accumulations momentanées de débauche signifiante qui sont l'éthos du festival: "C'est de l'amourgandise, de l'indigespérance" 24 , "ça lui a de plus en plus

21 À telle enseigne que, à l'intérieur de nombreux festivals (lesquels ont à l'ordinaire leur service d'ordre), une forme de contestation prend forme et une marge se sépare sous la forme de ces anti-festivals que sont les festivals off.

22 Un nom, d'obédiences shakespearienne et rimbaldienne, dont on remarquera surtout qu'il est un nom de la retombée, un nom de la chute. Chez Ducharme, l'oreille voit. C'est pourquoi "Bottom" laisse voir et entendre "tombeau".

23 S'il s'agissait de foumir une lecture sociocritique complète du roman, il faudrait nuancer ici fortement le propos. Mon but cependant n'est pas celui-là et se borne à faire dialoguer la ville et le roman ducharmien autour de la notion de festivalesque, proposée à des fins heuristiques.

24 Réjean Ducharme, Dévadé, Paris, Gallimard/Lacombe, 1990, p. 79. 
plu comme état" ${ }^{25}$, "la crucifiante cruciverbiste" 26 , "Pendant qu'elle ouvrait l'agrafe et testait le bijou sur ses doigts, je me prosternais sur ses pieds, j'aspirais leur moiteur en reniflant, j'enfonçais les dents dans la mie en grognant, chevrotant, roulant les râles les plus défaillants pour achever de la dégoûter, pour l'écœurer jusqu'à ce que ses os craquent. Elle était ravie! Elle avait fleuré ces talents qu'elle poussait au plein épanouissement. " 27 Ces explosions stylistiques ou rhétoriques ${ }^{28}$ caracolent autour d'une atmosphère festive puisque, de Bottom à Nicole, de la patronne à Juba, de Bruno au poète Adé, tout un petit monde évolue dans "l'atmosphère du temps des fêtes". La fête cependant n'a rien de grandiose, loin de là. Elle fait intimement corps avec une ville associée au vide: "Je me jette dans le vide où baigne la ville, et je marche "29, à la chute et à la descente: "Amuse-toi. Descends à Montréal" 30 , "Je descendais en ville, dans la force du mot" 31 , à la dégradation mêlée d'ivresse et de plaisir: "Notre bouteille de vodka nous avait fait traverser la ville en triomphe; le diable nous emportait ${ }^{32}$. Les individus qui la peuplent ou qui vivent dans ses sous-sols ou sur ses bordures y collectionnent des choses miteuses à l'instar de Bruno ou des objets d'art qui semblent devenus jetables, comme Nicole qui possède "tous les disques de Manitas de Plata." Annonçant en ces termes son petit festival de guitare personnel: "Il est né gitan, tu te rends compte!", elle se voit répondre: "- Ouais, ça a dû lui faire vachement plaisir de pas se ramasser rada... ${ }^{33}$ Et comme toujours chez Ducharme - c'est une autre trace de ce que je nomme le festivalesque -, toutes les actions des personnages sont empêtrées dans une jubilation grotesque

25 Ibid., p. 142.

26 Ibid., p. 122.

27 Ibid., p. 140.

28 L'explosion, ou devrait-on dire l'implosion?, est l'un des rêves de Bottom: "Le clignotement des gyrophares jouant à fond sur mes nerfs, je me retiens mal de foncer, régler mon cas, passer une fois pour toutes à travers tout ce qui me retient... Il faut exploser tout de suite en un seul coup, ou en mille miettes le long du long détour, en rattrapant le Boulevard par le chemin du lac des Quatorze-Iles..." (Ibid., p. 125).

29 Ibid., p. 232.

30 Ibid., p. 136.

31 Ibid., p. 105. La descente en ville n'est jamais loin d'une petite descente en enfer: "Pas Seigneur: Bottom. En enfer, on mappelle Bottom." (Ibid., p. 124).

32 Ibid., p. 71.

33 Ibid., p. 217. 
126

générale ${ }^{34}$, dans des problèmes d'argent perpétuels, dans une rage de consommer jusqu'à l'abrutissement qui culmine dans l'ingurgitation répétitive de moultes canettes par Bottom. Enfin, d'excellentes lectures de Ducharme l'ont amplement démontré, le roman ducharmien se présente comme un festival de citations littéraires et de références culturelles ${ }^{35}$.

Ces éléments de description font apparaître que le festivalesque entrelace plusieurs cordes, dont les trois suivantes: une activité et une dimension culturelles qui s'expriment par gerbes, par saccades, par séquences; une consommation ostentatoire de produits divers, lesquels relèvent plus ou moins tous, chez Ducharme, du prêt-à-consommer (une sorte de greasy-life, si l'on veut); un consentement à l'état des lieux, un abandon résolu de toute tentative pour régénérer quoi que ce soit et surtout pas "le monde". De l'intrication de ces trois éléments peuvent (entre autres) se dégager deux caractéristiques du rapport de l'écriture festivalesque à la ville.

Il y a d'abord que ce consentement à l'état des lieux, qui est aussi à la fois un consentement à la Montréal contemporaine, à ses perpétuelles modifications, à son enlaidissement têtu, et un consentement au capitalisme tel qu'il se vit en terre québécoise, c'est-à-dire avec une grande et constante et matérialiste et très pragmatique satisfaction, il ne faut pas croire que l'esthétique ducharmienne se contente de l'imposer ou de le reproduire. Elle l'ironise et le sémantise sous l'angle d'un "subir et transgresser "36, sur le mode d'un "hélas positif " ${ }^{37}$ que le sujet adopte comme on

34 En ce sens, le festivalesque ducharmien n'est nullement la conséquence d'un festival de la gastronomie, mais plutôt son négatif: "Je suis inséré. J'ai un job!... Sous aide-cuisinier dans le greasy-food." (Ibid., p. 235). À mes yeux, ces lignes de Pierre Nepveu, quoique destinées à Gilbert Langevin, s'appliquent tout à fait au grotesque ducharmien: "Le grotesque n'est pas une fête carnavalesque mais l'activation d'un sujet solitaire dans un réel mourant, un choix pour la sainteté démoniaque, pour l'angélisme noir." (Lécologie du réel, p. 122).

35 Voir Gilles Marcotte, Le roman à limparfait. Essais sur le roman québécois diajourd hui, Montréal, La Presse, 1976, p. 57-92.

36 Réjean Ducharme, op. cit., p. 130.

37 Vaguement proche de celui que "la patronne" enseigne à Bottom: "Elle m'explique qu'on reconnât les Italiens à leur mágari, cet hélas positif, tout joyeux de bienveillance, qu'ils emploient à tout propos, sinon à tout hasard, sans autre besoin que de produire un bel objet emotif, une forme sonore de vitalité." (Ibid, , p. 121). 
le ferait d'une armure, car il n'y a que dans ce dédain protégé, fidèle à celui qui permettait à Rimbaud d'avoir raison de s'évader ${ }^{38}$, qu'il puisse trouver un espace de liberté propice à accueillir son langage, son amour et son désir.

Il y a ensuite que les rafales et les gerbes d'étincelles littéraires et culturelles parsemées dans le roman ducharmien surviennent dans et sur une ville énergiquement vide. Je crois que l'on peut lire dans cette connexion la transposition imaginaire d'une réalité montréalaise que les travaux du groupe de recherche "Montréal imaginaire" n'ont cessé de rencontrer sur leur chemin ${ }^{39}$. La culture, la littérature ne sont pas acquises à Montréal, elles ne semblent pas aller de soi, elles n'éprouvent pas pour se développer le socle d'une tradition ferme et, quand tradition il y a, elle ne s'avère guère très marquée par l'urbanité. La multiplication des festivals à Montréal, en ses meilleures réalisations, est la façon dont les Montréalais ont négocié avec cette difficulté, leur façon de célébrer, de combler et de dépasser un manque. Et le festivalesque, celui de Ducharme ou d'autres écrivains - car je crois que l'on pourrait étendre la portée de cette notion à d'autres écritures, contemporaines ou antérieures (je songe notamment à Hector Berthelot ou à Claude Gauvreau) - , est une manière littéraire de donner sens à cette alliance de manque et de comble, de frustration et de plaisir. Comme le dit André le narrateur-délégué de L'biver de force, résumant en deux petites phrases tout ce que j'ai pu dire depuis le début de cette communication: "On mange puis on se cultive. Qu'est-ce qu'on peut demander de plus? 40

38 Voir l'épigraphe.

39 Cf. Pierre Nepveu et Gilles Marcotte (dir.), Montréal imaginaire. Ville et littérature, Montréal, Fides, 1992, $424 \mathrm{p}$.

40 Réjean Ducharme, L'biver de force, p. 96. 